PROCEEDINGS OF THE

AMERICAN MATHEMATICAL SOCIETY

Volume 131, Number 4, Pages 1267-1271

S 0002-9939(02)06671-6

Article electronically published on September 5, 2002

\title{
THE NEVANLINNA COUNTING FUNCTIONS FOR RUDIN'S ORTHOGONAL FUNCTIONS
}

\author{
TAKAHIKO NAKAZI
}

(Communicated by Juha M. Heinonen)

\begin{abstract}
H^{\infty}$ and $H^{2}$ denote the Hardy spaces on the open unit disc $D$. Let $\phi$ be a function in $H^{\infty}$ and $\|\phi\|_{\infty}=1$. If $\phi$ is an inner function and $\phi(0)=0$, then $\left\{\phi^{n} ; n=0,1,2, \cdots\right\}$ is orthogonal in $H^{2}$. W. Rudin asked if the converse is true and C. Sundberg and C. Bishop showed that the converse is not true. Therefore there exists a function $\phi$ such that $\phi$ is not an inner function and $\left\{\phi^{n}\right\}$ is orthogonal in $H^{2}$. In this paper, the following is shown: $\left\{\phi^{n}\right\}$ is orthogonal in $H^{2}$ if and only if there exists a unique probability measure $\nu_{0}$ on $[0,1]$ with $1 \in \operatorname{supp} \nu_{0}$ such that $N_{\phi}(z)=\int_{|z|}^{1} \log \frac{r}{|z|} d \nu_{0}(r)$ for nearly all $z$ in $D$ where $N_{\phi}$ is the Nevanlinna counting function of $\phi$. If $\phi$ is an inner function, then $\nu_{0}$ is a Dirac measure at $r=1$.
\end{abstract}

\section{INTRODUCTION}

Let $D$ be the open unit disc in the complex plane $\mathbf{C}$ and $H^{p}, 1 \leq p \leq \infty$, the usual Hardy spaces on $D$. Any function $f$ in $H^{p}$ has a boundary value on $\partial D$ almost everywhere with respect to the Lebesgue measure $d \theta / 2 \pi$. It may be assumed that $H^{p}$ is a closed subspace in the usual Lebesgue space $L^{p}=L^{p}(\partial D, d \theta / 2 \pi)$. Hence $H^{2}$ is a Hilbert space.

If $\phi$ is an inner function in $H^{\infty}$, that is, $|\phi|=1$ a.e. $d \theta / 2 \pi$ on $\partial D$ and $\phi(0)=0$, then $\left\{\phi^{n} ; n=0,1,2, \cdots\right\}$ is orthogonal in $H^{2}$. The following problem was posed by W.Rudin in 1988 (at an MSRI conference) (see [2]): If $\phi$ is a function in $H^{\infty}$ with $\|\phi\|_{\infty}=1$ such that $\left\{\phi^{n} ; n=0,1,2, \cdots\right\}$ is orthogonal in $H^{2}$, must $\phi$ be an inner function? C.Sundberg $[8$ and C.Bishop 1 independently solved the problem. In fact, they showed that there exists a function $\phi$ such that $\phi$ is not an inner function and $\left\{\phi^{n}\right\}$ is orthogonal in $H^{2}$. We are still interested in a function $\phi$ in $H^{\infty}$ such that $\left\{\phi^{n}\right\}$ is orthogonal in $H^{2}$ because such a function which is not an inner function is mysterious. For a function $\phi$ in $H^{\infty}$ with $\|\phi\|_{\infty}=1$, we call it a Rudin's orthogonal function when $\left\{\phi^{n} ; n=0,1,2, \cdots\right\}$ is orthogonal in $H^{2}$.

For a function $\phi$ in $H^{\infty}$ with $\|\phi\|_{\infty}=1$, the Nevanlinna counting function of $\phi, N_{\phi}$, is defined on $D \backslash\{\phi(0)\}$ by

$$
N_{\phi}(w)=\sum_{\phi(z)=w} \log \frac{1}{|z|}
$$

Received by the editors December 22, 2000 and, in revised form, December 6, 2001.

2000 Mathematics Subject Classification. Primary 30D50.

This research was partially supported by Grant-in-Aid for Scientific Research, Ministry of Education. 
where multiplicities are counted and $N_{\phi}(w)$ is taken to be zero if $w$ is not in the range of $\phi$. P.S.Bourdon [2] used the Nevanlinna counting function to attack Rudin's orthogonality problem. In fact, he showed the following are equivalent: (a) $\left\{\phi^{n} ; n=0,1,2, \cdots\right\}$ is orthogonal in $H^{2} ;(b)$ there is a non-negative function $g$ on $[0,1]$ such that for almost every $r \in[0,1], N_{\phi}\left(r e^{i \theta}\right)=g(r)$ for almost every $\theta \in[0,2 \pi]$.

In this paper, using a representing measure of a moment sequence we show the following: $\left\{\phi^{n} ; n=0,1,2, \cdots\right\}$ is orthogonal in $H^{2}$ if and only if there exists a unique positive Borel measure $\nu_{0}$ such that $1 \in \operatorname{supp} \nu_{0}, \nu_{0}([0,1])=1$ and

$$
N_{\phi}(z)=\int_{|z|}^{1} \log \frac{r}{|z|} d \nu_{0}(r)
$$

for nearly all $z$ in $D$.

\section{Result}

Let $\phi$ be a function in $H^{\infty}$ with $\|\phi\|_{\infty}=1$. For an analytic function $f$ on $D, C_{\phi} f(z)=f(\phi(z))(z \in D)$. When $\nu$ is a positive Borel measure on $\bar{D}$ and $\nu(\bar{D})=1, H^{2}(\nu)$ denotes the closure of all analytic polynomials in $L^{2}(\nu)$.

Lemma 1. Suppose $\phi$ is a function in $H^{\infty}$ with $\|\phi\|_{\infty}=1$. If $\left\{\phi^{n} ; n=0,1,2, \cdots\right\}$ is orthogonal in $\mathrm{H}^{2}$, then there exists a unique radial Borel measure $\nu$ such that $C_{\phi}$ is an isometric operator from $H^{2}(\nu)$ into $H^{2}$ where $d \nu=d \nu_{0}(r) d \theta / 2 \pi$ and $1 \in \operatorname{supp} \nu_{0}$.

Proof. Suppose $\left\{\phi^{n}\right\}$ is orthogonal in $H^{2}$. Let $\mathcal{H}_{\phi}^{2}$ be the closure of all polynomials of $\phi$ in $H^{2}$. Put

$$
\beta(n)=\left(\int_{0}^{2 \pi}|\phi|^{2 n} d \theta / 2 \pi\right)^{1 / 2} \quad(n=0,1,2, \cdots) .
$$

$\mathcal{H}^{2}(\beta)$ denotes the set of all power series: $f(z)=\sum_{n=0}^{\infty} \hat{f}(n) z^{n}$ with $\|f\|_{\beta}=$ $\sum_{n=0}^{\infty}|\hat{f}(n)|^{2} \beta(n)^{2}<\infty . \mathcal{H}^{2}(\beta)$ is a Hilbert space with the inner product: $(f, g)=$ $\sum_{n=0}^{\infty} \hat{f}(n) \overline{\hat{g}(n)} \beta(n)^{2}$. Then $\mathcal{H}_{\phi}^{2}$ is isometrically isomorphic to $\mathcal{H}^{2}(\beta)$ where

$$
F=\sum_{n=0}^{\infty}\left(\int_{0}^{2 \pi} F \bar{\phi}^{n} d \theta / 2 \pi\right) \phi^{n} \rightarrow f=\sum_{n=0}^{\infty} \hat{f}(n) z^{n}
$$

The multiplication operator $M_{z}$ on $\mathcal{H}^{2}(\beta)$ by $z$ is injective and subnormal because the multiplication operator $M_{\phi}$ on $\mathcal{H}_{\phi}^{2}$ is $\operatorname{subnormal.} \sup _{n}|\beta(n+1) / \beta(n)|=1$ because $\beta(n) \geq \beta(n+1)$ and so $\left\|M_{z}\right\|=1$ (see [7. p. 59]). It is known [7, Proposition $25]$ that $\mathcal{H}^{2}(\beta)$ is isometrically isomorphic to $H^{2}(\nu)$ where $d \nu=d \nu_{0}(r) d \theta / 2 \pi$ and $\nu_{0}$ is a Borel probability measure on $[0,1]$ with $1 \in \operatorname{supp} \nu_{0}$. Thus $\mathcal{H}_{\phi}^{2}$ is isometrically isomorphic to $H^{2}(\nu)$ and so $C_{\phi}$ is an isometric operator from $H^{2}(\nu)$ into $H^{2}$. For the uniqueness of $\nu$, if there exists another radial measure $d \lambda=d \lambda_{0}(r) d \theta / 2 \pi$ such that $C_{\phi}$ is isometric from $H^{2}(\lambda)$ into $H^{2}$, then

$$
\int_{\bar{D}}|z|^{2 n} d \nu=\int_{\bar{D}}|z|^{2 n} d \lambda \quad(n=0,1,2, \cdots)
$$

and so $\int_{0}^{1} r^{2 n} d \nu_{0}=\int_{0}^{1} r^{2 n} d \lambda_{0}$ for $n=0,1,2, \cdots$. By the Müntz-Szasz theorem [6] Theorem 15.26], $\nu_{0}=\mu_{0}$ because $\sum_{n=1}^{\infty} 1 / 2 n=\infty$. Thus $\nu=\lambda$. 
Lemma 2. For nearly all $w$ in $D$,

$$
N_{\phi}(w)=\int_{0}^{2 \pi} \log \left|\frac{w-\phi\left(e^{i \theta}\right)}{1-\bar{w} \phi\left(e^{i \theta}\right)}\right| d \theta / 2 \pi-\log \left|\frac{w-\phi(0)}{1-\bar{w} \phi(0)}\right| .
$$

Proof. By the proof of Littlewood's inequality [6, p. 187], for $w \in D \backslash\{\phi(0)\}$,

$$
N_{\phi}(w)=\lim _{r \rightarrow 1} \int_{0}^{2 \pi} \log \left|\frac{w-\phi\left(r e^{i \theta}\right)}{1-\bar{w} \phi\left(r e^{i \theta}\right)}\right| d \theta / 2 \pi-\log \left|\frac{w-\phi(0)}{1-\bar{w} \phi(0)}\right| .
$$

By a generalization of a theorem of Frostman [5], the lemma follows.

Lemma 3. Suppose $\nu$ is a radial measure, that is, $d \nu=d \nu_{0}(r) d \theta / 2 \pi$. If $C_{\phi}$ is an isometric operator from $H^{2}(\nu)$ into $H^{2}$, then

$$
N_{\phi}(z)=\int_{|z|}^{1} \log \frac{r}{|z|} d \nu_{0}(r)
$$

for nearly all $z$ in $D$.

Proof. If $C_{\phi}$ is isometric, then for any $w \in D$,

$$
\int_{\bar{D}}\left|\frac{w-z}{1-\bar{w} z}\right|^{2 n} d \nu=\int_{0}^{2 \pi}\left|\frac{w-\phi}{1-\bar{w} \phi}\right|^{2 n} d \theta / 2 \pi \quad(n=0,1,2, \cdots) .
$$

It is elementary to see that $x=\sqrt{1-\left(1-x^{2}\right)}=\sum_{n=0}^{\infty} a_{n}\left(1-x^{2}\right)^{n}$ and that $\sum_{n=0}^{\infty}\left|a_{n}\right|\left(1-x^{2}\right)^{n}<\infty(0 \leq x \leq 1)$. Hence by Lebesgue's dominated convergence theorem

$$
\begin{aligned}
\int_{0}^{2 \pi}\left|\frac{w-\phi}{1-\bar{w} \phi}\right| d \theta / 2 \pi & =\int_{0}^{2 \pi} \sum_{n=0}^{\infty} a_{n}\left(1-\left|\frac{w-\phi}{1-\bar{w} \phi}\right|^{2}\right)^{n} d \theta / 2 \pi \\
& =\sum_{n=0}^{\infty} a_{n} \int_{0}^{2 \pi}\left(1-\left|\frac{w-\phi}{1-\bar{w} \phi}\right|^{2}\right)^{n} d \theta / 2 \pi \\
& =\sum_{n=0}^{\infty} a_{n} \int_{\bar{D}}\left(1-\left|\frac{w-z}{1-\bar{w} z}\right|^{2}\right)^{n} d \nu \\
& =\int_{\bar{D}} \sum_{n=0}^{\infty} a_{n}\left(1-\left|\frac{w-z}{1-\bar{w} z}\right|^{2}\right)^{n} d \nu=\int_{\bar{D}}\left|\frac{w-z}{1-\bar{w} z}\right| d \nu
\end{aligned}
$$

because $\left|\sum_{n=0}^{k} a_{n}\left(1-\left|\frac{w-\phi}{1-\bar{w} \phi}\right|^{2}\right)^{n}\right| \leq \sum_{n=0}^{\infty}\left|a_{n}\right|<\infty$ and $\left|\sum_{n=0}^{k} a_{n}\left(1-\left|\frac{w-z}{1-\bar{w} z}\right|^{2}\right)^{n}\right| \leq$ $\sum_{n=0}^{\infty}\left|a_{n}\right|<\infty$. Similarly, as $x^{2 \ell+1}=\sqrt{1-\left(1-x^{4 \ell+2}\right)}$ we can show that

$$
\int_{0}^{2 \pi}\left|\frac{w-\phi}{1-\bar{w} \phi}\right|^{2 n+1} d \theta / 2 \pi=\int_{\bar{D}}\left|\frac{w-z}{-\bar{w} z}\right|^{2 n+1} d \nu \quad(n=0,1,2, \cdots) .
$$

Thus for any $w \in D$,

$$
\int_{0}^{2 \pi}\left|\frac{w-\phi}{1-\bar{w} \phi}\right|^{n} d \theta / 2 \pi=\int_{\bar{D}}\left|\frac{w-z}{1-\bar{w} z}\right|^{n} d \nu \quad(n=0,1,2, \cdots) .
$$

Since $\log x=\log (1-(1-x))=-\sum_{n=0}^{\infty} \frac{(1-x)^{n}}{n}(0<x \leq 1), \sum_{n=0}^{k} \frac{(1-x)^{n}}{n}$ is increasing when $0 \leq x \leq 1$ and $\lim _{k \rightarrow \infty} \sum_{n=0}^{k} \frac{(1-x)^{n}}{n}=-\log x$. By Lebesgue's monotone convergence theorem, for any $w \in D$,

$$
\int_{\bar{D}} \log \left|\frac{w-z}{1-\bar{w} z}\right| d \nu=\int_{0}^{2 \pi} \log \left|\frac{w-\phi}{1-\bar{w} \phi}\right| d \theta / 2 \pi .
$$


Hence by Lemma 1 , for nearly all $w \in D$,

$$
\begin{aligned}
N_{\phi}(w) & =\int_{\bar{D}} \log \left|\frac{w-z}{1-\bar{w} z}\right| d \nu-\log |w| \\
& =\int_{0}^{1} d \nu_{0}(r)\left(\int_{0}^{2 \pi} \log \left|\frac{w-r e^{i \theta}}{1-\bar{w} r e^{i \theta}}\right| d \theta / 2 \pi-\log |w|\right)
\end{aligned}
$$

because $\phi(0)=0$ and $\nu_{0}([0,1])=1$. Then

$$
N_{\phi}(w)=\int_{|w|}^{1} \log \frac{r}{|w|} d \nu_{0}(r)
$$

for nearly all $w \in D$ because

$$
\int_{0}^{2 \pi} \log \left|\frac{w-r e^{i \theta}}{1-\bar{w} r e^{i \theta}}\right| d \theta / 2 \pi-\log |w|=\left\{\begin{array}{cc}
\log \frac{r}{|w|} & (|w| \leq r) \\
0 & (|w|>r)
\end{array}\right.
$$

Theorem 1. Suppose $\phi$ is a function in $H^{\infty}$ with $\|\phi\|_{\infty}=1$. Then the following are equivalent:

(1) $\left\{\phi^{n} ; n=0,1,2, \cdots\right\}$ is orthogonal in $H^{2}$.

(2) $N_{\phi}(z)=N_{\phi}(|z|)$ for nearly all $z$ in $D$.

(3) There exists a unique Borel probability measure $\nu_{0}$ on $[0,1]$ with $1 \in$ supp $\nu_{0}$ such that

$$
N_{\phi}(z)=\int_{|z|}^{1} \log \frac{r}{|z|} d \nu_{0}(r)
$$

for nearly all z in $D$.

Proof. $(1) \Rightarrow(3)$. By Lemma 1 , there exists a unique radial measure $\nu$ such that $C_{\phi}$ is an isometric operator from $H^{2}(\nu)$ into $H^{2}$ where $d \nu=d \nu_{0}(r) d \theta / 2 \pi$ and $1 \in \operatorname{supp} \nu_{0}$. By Lemma 3,

$$
N_{\phi}(z)=\int_{|z|}^{1} \log \frac{r}{|z|} d \nu_{0}(r)
$$

for nearly all $z \in D . \quad(3) \Rightarrow(2)$ is clear. $(2) \Rightarrow(1)$. By the Littlewood-Paley theorem (see [2]), for $n>m$,

$$
\begin{aligned}
\int_{0}^{2 \pi} \phi^{n} \bar{\phi}^{m} d \theta / 2 \pi & =2 n m \int_{D} z^{n-1} \bar{z}^{m-1} N_{\phi}(|z|) d A(z) \\
& =4 n m \int_{0}^{1} r^{n+m-1} N_{\phi}(r) d r \int_{0}^{2 \pi} e^{i(n-m) \theta} d \theta / 2 \pi=0 .
\end{aligned}
$$

\section{REFERENCES}

1. C. Bishop, Orthogonal functions in $H^{\infty}$, preprint

2. P. S. Bourdon, Rudin's orthogonality problem and the Nevanlinna counting function, Proc. Amer. Math. Soc. 125(1997), 1187-1192. MR 98b:30034

3. J. A. Cima, B. Korenblum and M. Stessin, On Rudin's orthogonality and independence problem, preprint

4. W. Rudin, Real and Complex Analysis, Third Edition, McGraw-Hill, Inc. MR 88k:00002

5. W. Rudin, A generalization of a theorem of Frostman, Math. Scand. 21(1967), 136-143. MR 38:3463 
6. J. H. Shapiro, Composition Operators and Classical Function Theory, Springer-Verlag. MR 94k:47049

7. A. L. Shields, Weighted shift operators and analytic function theory, Math. Surveys, Amer. Math. Soc. 13, 49-128. MR 50:14341

8. C. Sundberg, Measures induced by analytic functions and a problem of Walter Rudin, preprint Department of Mathematics, Hokkaido University, Sapporo 060-0810, Japan

E-mail address: nakazi@math.sci.hokudai.ac.jp 